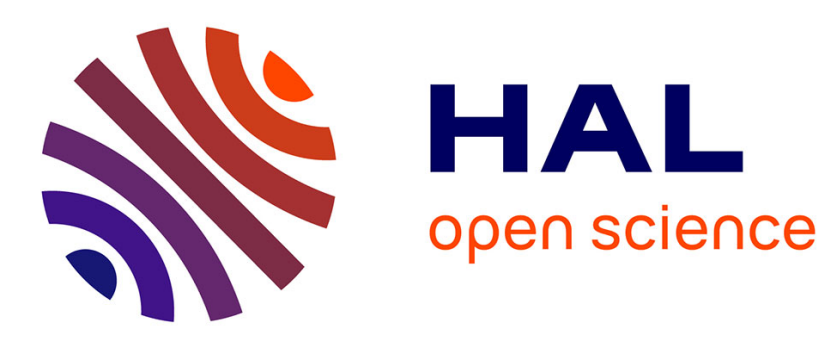

\title{
Decision under Uncertainty: the Classical Models
}

Alain Chateauneuf, Michèle Cohen, Jean-Yves Jaffray

\section{To cite this version:}

Alain Chateauneuf, Michèle Cohen, Jean-Yves Jaffray. Decision under Uncertainty: the Classical Models. 2008. halshs-00348818

\section{HAL Id: halshs-00348818 https://shs.hal.science/halshs-00348818}

Submitted on 22 Dec 2008

HAL is a multi-disciplinary open access archive for the deposit and dissemination of scientific research documents, whether they are published or not. The documents may come from teaching and research institutions in France or abroad, or from public or private research centers.
L'archive ouverte pluridisciplinaire HAL, est destinée au dépôt et à la diffusion de documents scientifiques de niveau recherche, publiés ou non, émanant des établissements d'enseignement et de recherche français ou étrangers, des laboratoires publics ou privés. 


\section{Documents de Travail du Centre d'Economie de la Sorbonne}

C

E

$\mathrm{S}$

W

0

1

k

i

n

g

$\mathrm{P}$

a

$\mathrm{p}$

e

$r$

$\mathrm{S}$

CENTRE NATIONAL

DE LA RECHERCHE

SCIENTIFIQUE

Maison des Sciences Économiques, 106-112 boulevard de L'Hôpital, 75647 Paris Cedex 13

http://ces.univ-paris1.fr/cesdp/CES-docs.htm

ISSN : 1955-611X 


\title{
Decision under Uncertainty: the Classical Models
}

\author{
Alain Chateauneuf*, Michèle Cohen* and Jean-Yves Jaffray** \\ *Paris School of Economics and CES-University Paris I Panthéon-Sorbonne \\ **Laboratoire d'Informatique de l'Université de Paris VI
}

December 3, 2008 


\section{Résumé:}

Ce chapitre d'ouvrage collectif a pour but de présenter les modèles classiques de décision dans l'incertain, c.a.d. dans des situations où les événements n'ont pas de probabilité "objective". Nous présentons successivement les deux principales théories, leur axiomatique, la justification et l'interprétation de leurs axiomes et leurs principales propriétés : d'abord, le modèle d'Espérance d'Utilité Subjective, dû à Savage (Savage, 1954), puis la théorie dûe à Anscombe-Aumann (Anscombe and Aumann, 1963), dans un cadre différent. Les deux théories imposent l'usage d'une représentation probabiliste. Nous discutons ensuite ces théories en liaison avec le résultat expérimental connu sous le nom de "Paradoxe d'Ellsberg ".

Mots clé: Incertain, Probabilité subjective, Espérance d'Utilité Subjective, Savage, Anscombe et Aumann, Paradoxe d' Ellsberg.

JEL: D81

\section{Abstract}

This chapter of a collective book is dedicated to classical decision models under uncertainty, i.e. under situations where events do not have "objective" probabilities with which the Decision Maker agrees. We present successively the two main theories, their axiomatic, the interpretation and the justification of their axioms and their main properties: first, the general model of Subjective Expected Utility due to Savage (Savage, 1954), second, the Anscombe-Aumann (1963) theory, in a different framework. Both theories enforce the universal use of a probabilistic representation. We then discuss this issue in connection with the experimental result known as the Ellsberg paradox.

Key words: Uncertainty, Subjective Probability, Subjective Expected Utility, Savage, Anscombe and Aumann, Ellsberg Paradox.

JEL: D81 


\section{Introduction}

This chapter is dedicated to classical decision models under uncertainty. Following KNIGHT (1921), the term risk is reserved to situations in which events have "objective" probabilities with which the DM agrees. This is typically the case in games of chance, such as card games, roulette, etc.; risk also encompasses all situations in which reliable statistical data are available. In addition to situations of risk, there seems to be a great variety of other situations of uncertainty that the DM can encounter: upper/lower probability intervals, possibilities/necessities, complete ignorance, small samples, etc.

Rather surprisingly, the classical models of decision making under uncertainty enforce the universal use of a probabilistic representation: every situation of uncertainty is identifiable to a situation of subjective risk. This means that every DM behaves "as if" he had probabilistic beliefs on all the events; on the other hand, these beliefs can vary from one DM to another.

The justification of this representation is based on axioms of "rational behavior". The most famous model giving an axiomatic justification of the Subjective Expected Utility (SEU) criterion is due to L.J. SAVAGE (1954): uncertainty reduces to subjective risk and decisions are ranked according to the expected utilities (EU) of their consequences.

Anscombe and Aumann (1963) also justify the SEU criterion, with a different model and in a different framework.

We present successively these two theories and insist on the interpretation and the justification of the axioms; we only provide an outline of the proofs.

\section{Subjective Expected Utility (SEU)}

\subsection{Definitions and notations}

$S$ is the set of states of nature, $\mathcal{E} \subset 2^{S}$ a set of parts of $S, A \in \mathcal{E}$ an event and $\mathcal{C}$ the set of consequences.

Decisions are identified to acts, which are applications from $S$ into $\mathcal{C}$; axiom $\mathbf{P} 8$ will introduce a "measurability" requirement. An act $f$ is a simple step act (resp. step act) when there exists a finite (resp. denumerable) partition $\left\{E_{i}, i \in I\right\}$ of $S$, with $E_{i} \in \mathcal{E}$ for every $i \in I$, such that $f\left(E_{i}\right)=\left\{c_{i}\right\}$ - a singleton. In particular, a constant act, $\delta_{c}$ is characterized by $\delta_{c}(S)=\{c\}$.

A graft is an operation which associates with two acts $f, g$ and an event $E$ a third act $h$ defined by: $h(s)=f(s)$ for $s \in E, h(s)=g(s)$ for $s \in E^{c}$; such an act is denoted by $h=f E g$.

Preferences are represented by binary relation $\succsim$ on the set of acts $\mathbb{V}$. 


\subsection{The SEU criterion}

Preferences $\succsim$ comply with Subjective Expected Utility (SEU) theory when they can be explained by the expected utility criterion, i.e. when there exist a (subjective) probability measure $P$ on the events and a utility function $u$ on the consequences such that, for all acts $f, g$ :

$$
f \succsim g \Longleftrightarrow \int_{S} u(f(.)) d P \geq \int_{S} u(g(.)) d P
$$

Savage (1954) and later Anscombe and Aumann (1963), have proposed axiomatic justifications of this criterion; Anscombe and Aumann, require a special structure for the consequence set, which is the set of all lotteries (finite support distributions) on the outcome set; they moreover assume the validity of the EU criterion for comparing these lotteries.

SAVAGE's framework is more general, and may seem more natural, since it does not require any particular structure on the consequence set; on the other hand, his approach is rather complex; Anscombe and AUmann have a simpler task, since they can take advantage of the properties of linear utility on the lotteries. We begin with the presentation of the theory of SAVAGE.

\section{The theory of Savage}

\subsection{Savage's axioms, their interpretation and some impli- cations}

N.B. The axiom system presented here is somewhat different from that of SAVAGE: it leads to a representation of the beliefs on the events by a $\sigma$-additive probability measure(as opposed to a simply additive probability in SAVAGE's).

\subsubsection{Preferences on the acts}

The first axiom postulates the existence of a rich structure on both the set of the events and the set of the acts and requires that the preference relation be a weak order (reflexivity, transitivity and completeness).

P1 Weak ordering of the acts

(i) The set of the events $\mathcal{E}$ is a $\sigma$-algebra;

(ii) The set of the acts $\mathbb{V}$ contains all step acts and is closed with respect the grafting operation.

(iii) $\succsim$ is a weak order on $\mathbb{V}$.

Conditions (i) and (ii) are technical; the structures imposed to $\mathcal{E}$ and $\mathbb{V}$ could not be dispensed with, even though they imply the existence of unrealistic acts (for instance of acts giving good consequences conditionally on unfavorable events); condition (iii) is standard in decision theory; note, though, that standard rationality arguments such as the avoidance of money pumps forbid 
preference cycles but cannot justify completeness.

The next axiom is the key axiom of SAVAGE's theory. It states that a common modification of the common part of two acts cannot modify the preference order between them.

\section{P2 Sure Thing Principle}

For all acts $f, g, h, h^{\prime}$ and for every eventE,

$$
f E h \succsim g E h \Longleftrightarrow f E h^{\prime} \succsim g E h^{\prime}
$$

Here again money pump arguments can only justify the following weakened version of P2:

$$
\mathbf{P}^{\prime} \mathbf{2} \quad f E h \succ g E h \Rightarrow f E h^{\prime} \succsim g E h^{\prime}
$$

This is an important remark for the following reason: backward induction is an efficient method for solving dynamic decision problems; it turns out, that its validity does not rely on $\mathbf{P} \mathbf{2}$ but only on $\mathbf{P}^{\prime} \mathbf{2}$; this opens the gate to alternative theories which, despite the fact they use different representations of uncertainty, remain operational.

Let us finally note that actual behavior often violates the Sure Thing principle P2 , and $\mathbf{P}^{\prime} \mathbf{2}$ as well; see below (section 2.3) the Ellsberg (1961) paradox; the AlLAis (1953) paradox can itself be presented as a violation of these axioms.

Induced preferences The axiom system will make it possible to derive from one primitive, preference relation $\succsim$ on the acts, several other binary relations, which will be interpreted as conditional preferences, preferences under certainty, preferences on the events, etc.

Conditional preferences given events For any event $E \in \mathcal{E}, \mathbf{P} 2$ allows one to define on the set of acts $\mathbb{V}$ a binary relation, preference given $E$, by:

$$
f \succsim_{E} g \Longleftrightarrow \text { for every } h, f E h \succsim g E h
$$

Relation $\succsim_{E}$ can be trivial, i.e. such that $f \succsim_{E} g$ for all $f, g \in \mathbb{V}$, in which case event $E$ is called a null event; in particular, $\emptyset$ is a null event.

Let us note that $\succsim_{E}$ only depends on the restrictions of $f$ and $g$ to $E$. This relation is generally interpreted, when $E$ is not null, as the expression of the DM's preferences conditionally to $E$, but this is only an interpretation (cf Ghirardato, 2002).

It is clear that, for every event $E \in \mathcal{E}, \succsim_{E}$ (preference given $E$ ) is a weak order. 
Preferences under certainty Preferences on acts $\succsim$ also induce preferences under certainty, $\succsim_{\mathcal{c}}$, by

$$
c^{\prime} \succsim_{\mathcal{C}} c^{\prime \prime} \Longleftrightarrow \delta_{c^{\prime}} \succsim \delta_{c^{\prime \prime}} \text { for all } c^{\prime}, c^{\prime \prime} \in \mathcal{C} .
$$

Relation $\succsim_{c}$ also is a weak order.

The introduction of $\succsim_{\mathcal{C}}$ is not interesting unless there exists intrinsic preferences under certainty, i.e. which do not depend on the information in the following sense:

P3 Existence of intrinsic preferences under certainty

For all consequences $c^{\prime}, c^{\prime \prime} \in \mathcal{C}$, for every non-null event $E \in \mathcal{E}$,

$$
c^{\prime} \succsim_{\mathcal{C}} c^{\prime \prime} \Longleftrightarrow \delta_{c^{\prime}} \succsim_{E} \delta_{c^{\prime \prime}}
$$

This axiom is more restrictive than it seems: for instance, it does not leave the possibility of expressing the influence on the ordering of consequences of an emotional trauma. Certain theories allow state dependent preferences, and do not require this axiome (see KARNI and SCHMEIDLER, 1993).

Preferences on the events We define next a preference relation on the events; for this, we shall use a particular class of step acts: the one-step acts.

Given $c^{\prime}, c^{\prime \prime} \in \mathcal{C}$ such that $c^{\prime} \succ_{\mathcal{C}} c^{\prime \prime}$, act $f_{A}$ offers prize $\left(c^{\prime} / c^{\prime \prime}\right)$ on $A$ when

$$
f_{A}(s)=c^{\prime} \text { if } s \in A, f_{A}(s)=c^{\prime \prime} \text { if } s \in A^{c}
$$

A preference relation $\succsim_{\mathcal{E}}$ on the set of events $\mathcal{E}$ can then be defined by:

For all $A, B \in \mathcal{E}, A \succsim_{\mathcal{E}} B \Longleftrightarrow$

there exists a prize $\left(c^{\prime} / c^{\prime \prime}\right)$ such that acts $f_{A}, f_{B}$ offering that prize on $A$ and

$$
B \text {, respectively, satisfy } f_{A} \succsim f_{B}
$$

The next axiom states that the value of the prize does not matter at all, which will make relation $\succsim_{\mathcal{E}}$ a weak order.

Its interpretation is the following: if, for a given prize, one prefers $f_{A}$ to $f_{B}$, it is because one believes $A$ to be more likely to obtain that $B$.

\section{P4 Non-influence of the prize}

For all consequences $c^{\prime}, c^{\prime \prime}, k^{\prime}, k^{\prime \prime} \in \mathcal{C}$ such that $c^{\prime} \succ_{\mathcal{C}} c^{\prime \prime}$ and $k^{\prime} \succ_{\mathcal{C}} k^{\prime \prime}$, for every act $f_{A}\left(\right.$ resp. $\left.f_{B}\right)$ offering prize $\left(c^{\prime} / c^{\prime \prime}\right)$ on event $A($ resp. $B) \in \mathcal{E}$, and every act $g_{A}\left(\right.$ resp. $\left.g_{B}\right)$ offering prize $\left(k^{\prime} / k^{\prime \prime}\right)$ on event $A$ (resp. $\left.B\right)$ :

$$
f_{A} \succsim f_{B} \Leftarrow \Rightarrow g_{A} \succsim g_{B}
$$


To prevent $\succsim_{\mathcal{E}}$ of being trivial, there must exist at least one feasible prize:

\section{P5 Non-triviality of preferences under certainty}

There exists consequences $c^{\prime}, c^{\prime \prime} \in \mathcal{C}$ such that $c^{\prime} \succ_{\mathcal{C}} c^{\prime \prime}$.

Note that $E$ is null if and only if $E \sim_{\mathcal{E}} \emptyset$.

Together, the preceding assumptions are sufficient to endow relation $\succsim \mathcal{E}$ with properties Q1 and Q2 of a qualitative probability (see below).

We shall need the following definition:

An event $A \succ_{\mathcal{E}} \emptyset$ is an atom (for $\succsim_{\mathcal{E}}$ ) when there is no event $B \subset A$ such that: $A \succ_{\mathcal{E}} B \succ_{\mathcal{E}} \emptyset$.

The next axiom will in particular imply that set $S$ is atomless (for $\succsim_{\mathcal{E}}$ ), a property which will prove to be crucial for the existence of a unique subjective probability. This axiom moreover implies continuity properties, related to those of the continuity axiom of linear utility theory.

\section{P6 Continuity}

For every pair of acts $f, g \in \mathbb{V}$ such that $f \succ g$, for every consequence $c \in \mathcal{C}$, there exists a finite partition $\left\{E_{i}, i \in I\right\}$ of $S$ such that, for all $i \in I$ :

(i) $f_{i} \succ g$ where $f_{i}(s)=f(s)$ for $s \notin E_{i}$ and $f_{i}(s)=c$ for $s \in E_{i}$;

(ii) $f \succ g_{i}$ whereg $g_{i}(s)=g(s)$ for $s \notin E_{i}$ and $g_{i}(s)=c$ for $s \in E_{i}$

This axiom can be interpreted as follows: if the modification of $f$ on $E_{i}$ cannot reverse preferences, then each $E_{i}$ must be judged sufficiently unlikely; one assumes thus: (i) the existence of partitions composed of arbitrarily unlikely events, which will imply the absence of atoms; and (ii) that every $f_{i}$ which is sufficiently close from $f$ (for the distance of weak convergence) must be ranked in the same way that $f$ with respect to $g$, which is a continuity property.

The last axiom in the original S AVAGE system is a dominance (or monotony) axiom:

\section{P7 Dominance}

For every event $E \in \mathcal{E}$,

(i) $f \succ_{E} \delta_{c}$ for every $c \in g(E)$ implies $f \succ_{E} g$;

(ii) $f \prec_{E} \quad \delta_{c}$ for all $c \in g(E)$ implies $f \prec_{E} g$

This axiom states that if one prefers $f$ to any consequence which can result from $g$, then one should prefer $f$ to $g$.

The last two axioms are not part of the original system of Savage; they will make sure that the subjective probability constructed is always $\sigma$-additive.

\section{P8 Measurability}

For every act $f \in \mathbb{V}$ and for every consequence $c \in \mathcal{C}$, sets $\left\{s \in S: f(s) \succsim_{\mathcal{C}}\right.$ c\} and $\{s \in S: f(s) \precsim c c\}$ belong to $\mathcal{E}$. 


\section{P9 Event-wise Continuity}

For all events $A, B \in \mathcal{E}$ and for every sequence of events $\left(A_{n}\right)_{n \in \mathcal{N}}$, if $A_{n} \downarrow A, A_{n} \succsim_{\mathcal{E}} B$, for every $n$, then $A \succsim_{\mathcal{E}} B$

\subsection{The steps of Savage's construction}

SAVAGE proves successively that:

(i) there exists, on the event set, qualitative probabilities and subjective (quantitative) probabilities which are compatible with them. Every act generates then a (subjective) probability measure on the consequence set.

(ii) preferences on the acts generate preferences on these probability measures, and these preferences satisfy the VON NEUMANN-M ORGENSTERN axioms for decision making under $\mathrm{risk}$.

\subsubsection{From qualitative probabilities to subjectives probabilities}

Existence of qualitative probabilities By definition, relation $\succsim_{\mathcal{E}}$ is a qualitative probability on $\mathcal{E}$, when it satisfies the following three properties:

Q1 $\succsim_{\mathcal{E}}$ is a weak order $S \succ_{\mathcal{E}} \emptyset$ and, for all $A \in \mathcal{E}, S \succsim_{\mathcal{E}} A \succsim_{\mathcal{E}} \emptyset$.

Q2 For all $A_{1}, A_{2}, B_{1}, B_{2}, \in \mathcal{E}$,

(i) $\left[A_{1} \cap A_{2}=\emptyset, A_{1} \succsim_{\mathcal{E}} B_{1}, A_{2} \succsim_{\mathcal{E}} B_{2}\right] \Longrightarrow A_{1} \cup A_{2} \succsim_{\mathcal{E}} B_{1} \cup B_{2}$;

(ii) $\left[A_{1} \cap A_{2}=\emptyset, A_{1} \succ_{\mathcal{E}} B_{1}, A_{2} \succsim_{\mathcal{E}} B_{2}\right] \Longrightarrow A_{1} \cup A_{2} \succ_{\mathcal{E}} B_{1} \cup B_{2}$.

Q3 For all $A, B \in \mathcal{E}$ and $\left(A_{n}\right)_{n \in \mathcal{N}}$,

$\left[A_{n} \downarrow A, A_{n} \succsim_{\mathcal{E}} B\right.$, for all $\left.n\right] \Longrightarrow A \succsim_{\mathcal{E}} B$.

The validity of properties Q1 and Q2 is a rather direct consequence of axioms $\mathbf{P 1}$ à $\mathbf{P 5}$. To establish the validity of Q2, the following intermediate property is useful:

$A_{1} \succsim_{\mathcal{E}} B_{1} \Longleftrightarrow A_{1} \cup E \succsim_{\mathcal{E}} B_{1} \cup E$ for every $E$ such that $E \cap\left[A_{1} \cup B_{1}\right]=\emptyset$

which is a straightforward consequence of $\mathbf{P} \mathbf{2}$.

Finally, property Q3 is just axiom P9.

Therefore relation $\succsim_{\mathcal{E}}$ is a qualitative probability on $\mathcal{E}$.

Remark 1 Conditions Q1 and Q2 are due to De Finetti (1937); VilleGAS (1964) added axiom Q3 in order to get a $\sigma$-additive version of subjective probability theory. 


\section{Existence of a compatible subjective probability}

A probability $P$ on $\mathcal{E}$ is compatible with $\succsim_{\mathcal{E}}$ if $P(A) \geqq P(B) \Longleftrightarrow A \succsim_{\mathcal{E}} B$.

It can be easily seen that conditions $\mathbf{Q 1}, \mathbf{Q} 2$ are necessary for the existence of a probability $P$ on $\mathcal{E}$ which is compatible with $\succsim \mathcal{E}$. A counter-example, due to Kraft, Pratt and Seidenberg (1959), shows that these conditions are not sufficient to insure the l'existence of a compatible probability . However, VILLEG AS (1967), by adding the assumption that set $\mathcal{E}$ is atomless, has obtained the following result:

When $\mathcal{E}$ is atomless, $Q 1, Q 2$, and $Q 3$ are sufficient conditions for the existence of a unique subjective probability, $P$, on $(S, \mathcal{E})$ compatible with qualitative probability $\succsim_{\mathcal{E}}$. Moreover:

(i) $E \in \mathcal{E}$ is null if and only if $P(E)=0$;

(ii) for every $E \in \mathcal{E}$ and every $\rho \in(0,1)$, there exists $A \subset E$ such that $P(A)=\rho P(E)$.

Here is an outline of VILLEGAS' proof:

Every event $A$ can be divided into two sub-events $A_{1}$ and $A_{2}$ satisfying $A_{1} \sim_{\mathcal{E}} A_{2}$; this implies in turn, by an inductive argument, that there exists a $2^{n}$-partition $\left\{E_{i}\right\}$ of the sure event $S$ with $E_{i} \sim_{\mathcal{E}} E_{j}$ for all $i, j$. Necessarily, a compatible probability $P$ is such that $P\left(E_{i}\right)=1 / 2^{n}$ for all $i$; moreover, probability $P(A)$ of an event $A$ of $\mathcal{E}$ such that $\underset{1 \leq i \leq k+1}{\cup} E_{i} \succ_{\mathcal{E}} A \succsim_{\mathcal{E}} \underset{1 \leq i \leq k}{\cup} E_{i}$, shall satisfy $P(A) \in\left[\frac{k}{2^{n}}, \frac{k+1}{2^{n}}[\right.$.

By taking the limit $(n \mapsto \infty), P(A)$ will be uniquely determined. Finally, one proves the additivity and the $\sigma$-additivity of $P$.

The preceding result can be interpreted roughly as follows: $\mathcal{E}$ contains all events linked to the outcomes of an arbitrary sequence of coin throws, where the coin used is believed to be unbiased by the DM. Thus to evaluate the probability of any given event, he only need to compare it with events, linked to the throwing sequence, to which he already attributes probabilities of the form $\frac{k}{2^{n}}$.

The absence of atoms in SAVAGE's axiom system comes essentially from P6 : for every non-null event $A$, one can find a partition $\left\{E_{i}\right\}$ such that $A \succ_{\mathcal{E}} E_{i} \succ_{\mathcal{E}} \emptyset$ for every $i$ and, in particular, $E_{i_{0}}$ such that $B=E_{i_{0}} \cap A$ satisfy $A \succ_{\mathcal{E}} B \succ_{\mathcal{E}} \emptyset$.

Together, SAVAGE's axioms imply both the absence of atoms and the validity of axioms Q1, Q2 and Q3, hence they imply the existence of a unique subjective probability on the events. We are back to a problem of decision making under risk - subjective risk here - but, as we shall see, the DM's behavior will not differ from his behavior under objective risk.

\subsubsection{Subjective lotteries and linear utility}

Now that a (unique) subjective probability $P$ has been constructed on the events, we can associate with every act $f$ the probability measure $P_{f}$ which it generates on consequence set $\mathcal{C}\left(P_{f}\right.$ is the image of $P$ by $\left.f\right)$. We note $\mathcal{L}_{0}$ the set of these probability measures. In particular, a simple step act generates 
a (subjective) lottery on $\mathcal{C}$, i.e. a probability measure with finite support; if the only feasible consequences of $f$ are the set $\left\{x_{i}, i=1, \ldots, n\right\}$, this set is the support of $P_{f}$ and $\left.P_{f}\left(x_{i}\right)=P\left(f^{-1}\left(x_{i}\right)\right), i=1, \ldots, n\right\}$.

One needs then to show that two acts generating the same lottery are necessarily indifferent for relation $\succsim$. This is a crucial step in the construction of SAVAGE, and also one of the most delicate ones. It basically exploits two properties: (i) the Sure Thing principle (P2); and (ii) the existence, for every event $A$ and every $\rho \in[0,1]$, of an event $B \subset A$ such that $P(B)=\rho P(A)$.

It results from $\mathbf{P} \mathbf{1}$ that $\mathcal{L}_{0}$ is the set of all lotteries on $\mathcal{C}$. There exists then, on lottery set $\mathcal{L}_{0}$, a preference relation, induced by the preferences existing on the acts, which we also denote by $\succsim$ :

$$
\begin{gathered}
P \succsim Q \Longleftrightarrow \text { there exist simple step acts } f \text { and } g \text { such that } \\
P_{f}=P, P_{g}=Q \text { and } f \succsim g
\end{gathered}
$$

Relation $\succsim$ on $\mathcal{L}_{0}$ is clearly a weak order,thus satisfying Axiom $\mathbf{1}$ of linear utility theory. The next step consists in proving that it moreover satisfies Axiom 2 (independence) and Axiom 3 (continuity).

The proof of these results confirms that P6 is indeed a continuity axiom (and not only a non-atomicity axiom); it also stresses the narrow links between P2 and A2; indeed, the Allais paradox constitutes a violation of both.

Thus the theorem of VON NEUMANN-Morgenstern applies to $\mathcal{L}_{0}$ : there exists on $\mathcal{L}_{0}$ a linear utility function $U$ and an associated vNM utility $u$. Returning to acts, we can therefore state:

The restriction to simple step acts of preference relation $\succsim$ can be explained by an expected utility criterion with respect to subjective probability measure $P$ on the events and utility function $u$ on the consequences; for two such acts $f$ and $g$ :

$$
\begin{gathered}
f \succsim g \Longleftrightarrow E u\left(P_{f}\right) \geqslant E u\left(P_{g}\right) \Longleftrightarrow \\
\sum_{i=1}^{n} P_{f}\left(\left\{x_{i}\right\}\right) u\left(x_{i}\right) \geqslant \sum_{j=1}^{n^{\prime}} P_{g}\left(\left\{y_{j}\right\}\right) u\left(y_{j}\right)
\end{gathered}
$$

It remains to extend the validity of the expected utility criterion from simple step acts to general acts.

\subsubsection{Extension of SEU to all acts}

SAVAGE's axiom P7 which concerns acts implies the validity of dominance axiom $\mathbf{A} 4$ for the probability measures these acts generate on $\mathcal{C}$. From which it follows that utility function $u$ above is bounded.

Moreover, by using $\mathbf{P 8}$, one can show that $u$ is also measurable so that the integral $\int_{S} u(f()) d P=.\int_{C} u(.) d P_{f}$ exists and has a finite value; then, its value can be associated with act $f$.

It still remains to show that the expected utility criterion is valid for preferences on the whole set of acts; first the extension from simple step acts (for which this 
criterion is straightforwardly valid) to generalized step acts is made by a similar reasoning to that used in EU theory. The extension to general acts follows; it uses the fact that every act is indifferent to a generalized step act.

One can then state SAVAGE's theorem :

\section{Theorem (Savage)}

Under axioms $\mathbf{P 1}$ to $\mathbf{P 9}$, preference relation $\succsim$ on $\mathbb{V}$ is representable by a utility function $U($.$) of the form:$

$$
U(.): f \mapsto \int_{S} u(f(.)) d P=\int_{C} u(.) d P_{f}
$$

- where $P($.$) is a ( \sigma$-additive) probability on the events of $E$;

- and $u($.$) is the von Neumann-Morgenstern utility on the probability set$ formed by the images of $P$ generated by the acts.

Moreover, $P$ is unique, whereas $U($.$) and u($.$) are unique up to a strictly$ increasing affine transformation.

The empirical validity of SAVAGE's model has serious limitations; the ALLA IS paradox does not only exhibit a pattern of behavior which is incompatible with EU under risk; this pattern is also incompatible with SEU under uncertainty.

There are other experiments, specific to uncertainty situations, where subjects display behavioral patterns which are incompatible with the existence of subjective probabilities (and a fortiori with SEU), such as the famous ELLSBERG paradox.

\subsection{The Ellsberg paradox}

EllSBERG (1961) describes the following situation: an urn contains 90 balls; 30 are red and 60 are blue or yellow, in unknown proportions; thus, there are $k$ blue balls and $(60-k)$ yellow balls, with unknown $k$ ranging from 0 to 60 . A random drawing of a ball from the urn will lead to the realisation of one of the events $R, B$ and $Y$, according to the color, Red, Blue or Yellow of the ball drawn.

ELLSBERG asks the subjects to choose between the following decisions: a bet on $(R)$ (decision $X_{1}$ ) or a bet on $(B)$ (decision $X_{2}$ ); and next, independently, to choose between: a bet on $(R \cup Y)\left(\right.$ decision $\left.X_{3}\right)$ or a bet on $(B \cup Y)\left(\operatorname{decision} X_{4}\right)$.

The following table gives the payments (in $\square$ ) associated with each decision fot each possible event:

\begin{tabular}{llll} 
& $R$ & $B$ & $Y$ \\
\hline & & & \\
$X_{1}$ & 100 & 0 & 0 \\
$X_{2}$ & 0 & 100 & 0 \\
$X_{3}$ & 100 & 0 & 100 \\
$X_{4}$ & 0 & 100 & 100 \\
& & & \\
\hline
\end{tabular}


Typically, a majority of subjects choose $X_{1}$ and $X_{4}$, thus revealing preferences: $X_{1} \succ X_{2}$ and $X_{4} \succ X_{3}$; this constitutes a violation of the Sure Thing principle P2: a modification on event $y$ of the common consequence $0 \square$ of $X_{1}$ and $X_{2}$, consisting in replacing it by a different common consequence $100 \square$ which transforms $X_{1}$ into $X_{3}$ and $X_{2}$ into $X_{4}$, should leave preferences unchanged, i.e. lead to $X_{3} \succ X_{4}$ whenever $X_{1} \succ X_{2}$.

Since they do not respect $\mathbf{P} \mathbf{2}$, these sujets cannot abide by the SEU criterion. As a matter of fact, their behavior is incompatible with the very existence of subjective probabilities $p_{R}, p_{B}, p_{Y}$ for elementary events $R, B, Y: X_{1} \succ X_{2}$ would imply $p_{R}>p_{B}$ whereas $X_{4} \succ X_{3}$ would imply $p_{B}+p_{Y}>p_{R}+p_{Y}$; a contradiction.

To represent the situation described by ElLSBERG, we have taken $S^{\prime}=$ $\{R, B, Y\}$ as set of states of nature and identified the bets with applications (acts) $X: S^{\prime} \rightarrow \mathbb{R}$.

We might have adopted another approach and taken a set of states of nature composed of 61 states, $S=\left\{s_{0}, s_{1}, \ldots, s_{k}, \ldots, s_{60}\right\}$, where a state $s_{k}$ corresponds to a given composition of the urn: " 30 red balls,$k$ blue balls, and $(60-k)$ yellow balls".

The decisions are then identifiable with applications from $S$ into $\mathcal{Y}$, the set of lotteries on $\mathcal{C}=\{0,100\}$ (i.e. the set of all probability measures on $\mathcal{C}$ which have a finite support).

Thus, the uncertain prospect offered by the decision giving a gain of $100 \square$ if the ball drawn is blue and of $0 \square$ otherwise, which, in the initial formalization, was described by act $X_{2}$, is now characterized by application $g_{2}$ associating with every state of nature $s_{k}$ of $S$ the corresponding lottery, $\left(0, \frac{90-k}{90} ; 100, \frac{k}{90}\right)$, i.e. the lottery giving a null gain with probability $\frac{90-k}{90}$ and a gain of $100 \square$ with probability $\frac{k}{90}$.

This is the framework adopted in the model of Anscombe and AumanN which we present below.

\section{The theory of Anscombe and Aumann}

The set of states of nature $S$ is finite. The algebra of events is $\mathcal{A}=2^{S}$.

We denote by $\mathcal{Y}$ the set of lotteries on an outcome set $\mathcal{C}$ (i.e. the set of all probability measures with finite support in $\mathcal{C}$ ). The set of acts $\mathbb{F}_{0}$ is then defined as the set of all applications from $S$ into $\mathcal{Y}$.

An act of this kind is called a "horse lottery" by reference to the sweepstake tickets, which offer different random gains (lottery tickets) depending on which 
horse wins the race.

In this model, consequences are not outcomes ( elements of $\mathcal{C}$ ) but lotteries on $\mathcal{C}$, which are elements of $\mathcal{Y}$. The set $\mathcal{Y}$ is a mixture set (see linear utility theory). By using this structure, one can define, for all $f$ and $h$ in $\mathbb{F}_{0}$ and every $\alpha$ in $[0,1]$, act $\alpha f+(1-\alpha) h$ by

$(\alpha f+(1-\alpha) h)(s)=\alpha f(s)+(1-\alpha) h(s)$ for every $s$ in $S$.

For this operation, $\mathbb{F}_{0}$ is itself a mixture set.

Preferences are defined by a weak order on set of acts $\mathbb{F}_{0}$, denoted by $\succsim$.

Relation $\succsim$ induces a preference relation (also denoted by $\succsim$ ) on the set of lotteries, by identifying a lottery $y$ of $\mathcal{Y}$ with the constant act $\delta_{y}$ in $\mathbb{F}_{0}$ (the act taking the same value, consequence $y$, for every $s$ in $S:)$

for all $y, z$ in $\mathcal{Y}, y \succsim z \Leftrightarrow \delta_{y} \succsim \delta_{z}$.

\subsection{The Anscombe-Aumann axiom system}

It consists in the following five axioms:

\section{AA1 Ordering axiom}

Preference relation $\succsim$ is a weak order on $\mathbb{F}_{0}$.

\section{AA2 Continuity axiom}

For all $X, Y, Z$ in $X_{0}$, satisfying $X \succ Y \succ Z$, there exist $\left.\alpha, \beta \in\right] 0,1[$ such that

$$
\alpha X+(1-\alpha) Z \succ Y \succ \beta X+(1-\beta) Z
$$

\section{AA3 Independence axiom}

For all $X, Y, Z$ in $\mathbb{F}_{0}$ and for every $\left.\left.\alpha \in\right] 0,1\right]$,

$$
X \succsim Y \Longleftrightarrow \alpha X+(1-\alpha) Z \succsim \alpha Y+(1-\alpha) Z
$$

\section{AA4 Monotony axiom}

For all $X, Y$ in $\mathbb{F}_{0},[X(s) \succsim Y(s)$, for every $s \in S] \Rightarrow X \succsim Y$

AA5 non-triviality of preferences

There exists at least one pair of acts $X, Y$ such that $X \succ Y$ 


\subsection{Comments and discussion}

The introduction, among the primitive concepts, of a set of lotteries $\mathcal{Y}$ presupposes the existence of "exogeneous "probabilities, i.e. which bear no relation with the beliefs of the DM; this was not the case with the model of SAVAGE, in which the existence of a probability is always a result and never an assumption.

In the model of ANSCOMBE and AUMANN, the outcome of an act is determined in two steps: during the first step, the uncertainty about the states of nat ure is resolved and the true state identified; during the second step, the lottery associated with this state is resolved, and the final outcome determined. One of the important points of the proof of the representation theorem will consist in showing that the order of resolution of the two kinds of uncertainty is irrelevant for the DM.

The mixture set structure of $\mathbb{F}_{0}$ suggests to use formally the axioms of vON Neumann and Morgenstern. However, the acts of $\mathbb{F}_{0}$ being more complex than probability measures, these axioms acquire a wider significance; in particular, in this framework, the independence axiome implies, in the presence of the other axioms, the validity of SAVAGE's Sure Thing principle.

\subsection{The Anscombe-Aumann representation theorem}

\section{Theorem (Anscombe and Aumann)}

Under axioms $\mathbf{A A 1}$ to $\mathbf{A A 5}$, preference relation $\succsim$ on $\mathbb{F}_{0}$ is representable by a utility function,

$$
V(.): X \mapsto \sum_{s \in S} U(X(s)) P(\{s\})
$$

where $P($.$) is an aditive probability measure on (S, \mathcal{E})$, and $U($.$) is the linear utility function on \left(\mathcal{L}, \succsim_{\mathcal{L}}\right)$.

$P$ is unique and $V($.$) , like U($.$) , is unique up to a strictly increasing linear$ transformation.

In this theory, every type of uncertainty is reducible to subjective risk, and the criterion under risk - under objective risk (lotteries) as under subjective risk - is the EU criterion.

The proof has several steps.

First, by restricting axioms AA1-AA3 to the constant acts $\delta_{y}$, identified with lotteries $y$, one remarks that VON NEUMANN-Morgenstern theory applies to these constant acts; then, it is easily seen that the following representation is valid for general acts:

$$
X \succsim Y \Longleftrightarrow U(X) \geqslant U(Y) \text { with } U(X)=\sum_{s \in S} U_{s}(X(s))
$$


where each $U_{s}$ is a linear utility, i.e. where $U_{s}(X(s))$ is the expectation of a utility function $u_{s}$ with respect to lottery $X(s)$.

The preceding linear utilities $U_{s}$ depend on state $s$.

Now, by taking into account axioms AA4-AA5, one can show that the $U_{s}$ are in fact proportional and that there exists thus a unique probability measure $P$ such that ratio $\frac{U_{s}}{P(\{s\})}$ is independent of $s$. It suffices then to set $\frac{U_{s}}{P(\{s\})}=U$ to obtain $U(X)=\sum_{s \in S} U(X(s)) P(\{s\})$.

Remark 2 In the ANSCOMBE-AUMANN framework, since $S$ is finite, the question of the $\sigma$-additivity of probability $P$ is pointless.

Remark 3 Fishburn (1970) has extended Anscombe-Aumann theory to an infinite set of states of nature ; in his extension subjective probability $P$ is only finitely additive.

\subsection{Back to the Ellsberg paradox}

Let us show now that the ELLSBERG paradox can be interpreted in the ANS COMBE and A Umann theory framework as a violation of the independence axiom AA3:

Let us use formalization $S=\left\{s_{0}, s_{1}, \ldots, s_{k}, \ldots, s_{60}\right\}$, where state $s_{k}$ corresponds to urn content: " 30 red balls, $k$ blue balls, and $60-k$ yellow balls".

The uncertain prospect described by act $X_{i}$ in SAVAGE's framework is now characterized, in the framework of ANSCOMBE and AumAnN, by mapping $g_{i}$ associating with each state of nature $s_{k}$ in $S$ a a lottery as indicated in the following table: 


\begin{tabular}{ll}
\hline$g_{1}\left(\approx X_{1}\right)$ & $\left(0, \frac{60}{90} ; 100, \frac{30}{90}\right)$ \\
$g_{2}\left(\approx X_{2}\right)$ & $\left(0, \frac{90-k}{90} ; 100, \frac{k}{90}\right)$ \\
$g_{3}\left(\approx X_{3}\right)$ & $\left(0, \frac{k}{90} ; 100, \frac{90-k}{90}\right)$ \\
$g_{4}\left(\approx X_{4}\right)$ & $\left(0, \frac{30}{90} ; 100, \frac{60}{90}\right)$ \\
$\delta_{0}$ & $\left(0, \frac{90}{90}\right)$ \\
$f$ & $\left(0, \frac{30+k}{90} ; 100, \frac{60-k}{90}\right)$ \\
$\frac{1}{2} g_{1}+\frac{1}{2} f=\frac{1}{2} g_{3}+\frac{1}{2} \delta_{0}$ & $\left(0, \frac{90+k}{180} ; 100, \frac{90-k}{180}\right)$ \\
$\frac{1}{2} g_{2}+\frac{1}{2} f=\frac{1}{2} g_{4}+\frac{1}{2} \delta_{0}$ & $\left(0, \frac{120}{180} ; 100, \frac{60}{180}\right)$ \\
\hline
\end{tabular}

The 4 first lines of the table indicate what becomes of acts $X_{1}, X_{2}, X_{3}, X_{4}$ in this new framework.

One can note that acts $g_{1}, g_{4}, \delta_{0}$ are now constant acts.

Axiom AA3 and the equalities of mixtures of acts in the last two lines of the table imply that:

$$
\begin{gathered}
g_{1} \succ g_{2} \Leftarrow \Rightarrow \frac{1}{2} g_{1}+\frac{1}{2} f \succ \frac{1}{2} g_{2}+\frac{1}{2} f \\
\Longleftrightarrow \frac{1}{2} g_{3}+\frac{1}{2} \delta_{0} \succ \frac{1}{2} g_{4}+\frac{1}{2} \delta_{0} \Longleftrightarrow g_{3} \succ g_{4}
\end{gathered}
$$

Thus choices $g_{1}$ and $g_{4}$ in the experiment are indeed a violation of AA3.

\section{Conclusion}

The possibility of justifying, by rationality arguments, the most crucial axioms of the theories of SAVAGE and ANSCOMBE andAUMANN has secured to the SEU model the rank of dominant normative model. Moreover, its use being simple, SEU has become a major tool in economic theory as well as in domains of application as diverse as insurance, finance, management, health care, and environment.

On the other hand, the limitations of SEU as a descriptive model, i.e. its incapacity to take into account fairly common behavior (ELLSBERG's paradox), may create difficulties in applications: for instance, when assessing subjective probabilities or constructing the utility function. 
There exists in the literature other models, which are more flexible than SEU, and can look quite different from it. Nonetheless, the two axiom systems which we have presented have invariably been the source of inspiration of those of the alternative "new" theories.

\section{References}

[1] Allais, M. "Le comportement de l'homme rationnel devant le risque: critique des postulats and axiomes de l'école américaine", Econometrica, 21, 503-546, 1953.

[2] Anscombe, F. and Aumann, R. "A definition of subjective Probability", Annals of Mathematical Statistics, 34, 199-205, 1963.

[3] de Finetti, B. "La prévision : ses lois logiques, ses sources subjectives". Annales de l'Institut Henri Poincaré, 7 ni1, p. 1-68, 1937.

[4] Debreu, G. "Representation of a preference ordering by a numerical function", in Decision Processes, Thrall, Coombs and Davies (eds), p159-165, Wiley, 1954.

[5] Ellsberg, D." Risk, Ambiguity, and the Savage Axioms", The Quarterly Journal of Economics, p643-669, 1961.

[6] Fishburn, P. Utility Theory for Decision Making. Wiley, 1970.

[7] Fishburn, P. The Foundations of Expected Utility. Reidel, 1982.

[8] Fishburn, P. and Wakker, P. " The invention of the independence condition for preferences", Management Science, 41, 1130-1144, 1995.

[9] Ghirardato, P. "Revisiting SAvage in a conditional world," Economic Theory, Springer Berlin Heidelberg, vol. 20(1), p 83-92, 2002.

[10] Jaffray, J.Y. Théorie de la Decision, Polycopié, Université de Paris VI, 1978.

[11] Jaffray, J.Y. "Choix séquentiels et rationalité" in Th. Martin (ed) Probabilités subjectives et rationalité de l'action, 27-36, CNRS Philosophie, 2003.

[12] Karni, E. and D. Schmeidler. "On the Uniqueness of Subjective Probabilities," Economic Theory, Springer Berlin Heidelberg, vol. 3(2), p 267-77, 1993.

[13] Knight, F. Risk, Uncertainty and Profit. Houghton Miffin, 1921.

[14] Kraft, C.H., Pratt, JW. and Seidenberg, A. "Intuitive probability on finite sets", Annals of Mathematical Statistics, 30, 408-419, 1959. 
[15] Kreps, D. Notes on the theory of choice, Underground classics in economics. Westview Press, 1988.

[16] Savage, L. The Foundations of Statistics, Dover, 1954.

[17] Villegas, C. "On qualitative probability $\sigma$-algebras". Annals of Mathematical Statistics, 35, p1787-1796, 1964.

[18] von Neumann, J. and Morgenstern, O. Theory of Games and Economic Behavior, Princeton University Press, Princeton, N.J., 1947.

[19] Anonymous : http://cepa.newschool.edu/het/essays/uncert/anscombe.htm, The Anscombe-Aumann Approach. 